\title{
PAPER
}

\section{Long term risks of stroke, myocardial infarction, and vascular death in "low risk" patients with a non-recent transient ischaemic attack}

\author{
T G Clark, M F G Murphy, P M Rothwell
}

See end of article for authors' affiliations .....................

Correspondence to: Dr P M Rothwell, Department of Clinical Neurology, Radcliffe Infirmary, Woodstock Road, Oxford, OX2 6HE, UK;

peter.rothwell@ clneuro.ox.ac.uk

Received 19 August 2002

In revised form

23 December 2002

Accepted

24 December 2002

\begin{abstract}
Background: Previous studies of prognosis after a transient ischaemic attack (TIA) have recruited patients soon after the event, when the risk of stroke is very high. However, the majority of patients survive for many years after a TIA, and the need for continued preventive treatment to lower vascular risk will need to be reassessed at a later date.

Obective: To determine the long term risks of stroke and other vascular events in patients with TIA who survive the initial high risk period.

Methods: 290 patients were studied who had initially been followed up after a TIA in the Oxford community stroke project and in a contemporaneous hospital based cohort study, and who were alive and stroke-free at the end of planned follow up in 1988. All patients were followed for a further 10 years, and the risks of major vascular events (stroke, myocardial infarction, vascular death) were determined. Standardised mortality ratios (SMR) were calculated from the observed numbers of fatal events and the number expected on the basis of age and sex in the general population.

Results: Median time since last TIA was 3.8 years (interquartile range, 2.2 to 5.8 years). The risk of major vascular events was constant through time. The 10 year risk of first stroke was $18.8 \% 195 \%$ confidence interval (CI), 13.6 to $23.7 ; 45$ events). The 10 year risk of myocardial infarction or death from coronary heart disease was $27.8 \%(95 \% \mathrm{Cl}, 21.8$ to $33.3 ; 67$ events) and there was a significant excess of fatal coronary events compared with that expected in the general population (SMR = 1.47; $95 \% \mathrm{Cl}, 1.10$ to $1.93 ; p=0.009$ ). A total of 114 patients had at least one major vascular event, with a 10 year risk of any first stroke, myocardial infarction, or vascular death of $42.8 \%(95 \% \mathrm{Cl}, 36.4$ to 48.5).

Conclusions: The overall risk of major vascular events remains high for 10 to 15 years after a TIA. It is important therefore that preventive treatments are continued in the long term, even in apparently "low risk" patients who have already survived free of stroke for several years.
\end{abstract}

! $\mathrm{t}$ is important to have accurate and reliable data on the prognosis of patients with vascular disease so that preventive treatments can be targeted effectively. ${ }^{12}$ Several studies have sought to provide these data for patients with transient ischaemic attacks (TIA). ${ }^{3-6}$ However, all of these studies recruited patients soon after the initial TIA, when the risk of stroke is very high. In routine clinical practice, many patients do not seek medical attention immediately after a TIA, $^{7}$ and the diagnosis is only made when they consult a physician for other reasons, often many months or even years later. Clinicians need to know the risks of stroke and other vascular events at that time.

In addition, as the majority of TIA patients survive for many years after the event, vascular risk will usually need to be reassessed at a later date, irrespective of when they presented. Indeed, this is now a requirement of the National Service Framework for Older People in the United Kingdom, which obliges all general practices to set up registers of patients with a previous TIA or stroke. ${ }^{8}$ It is intended that this will ensure that patients receive appropriate preventive treatment. However, given that the risk of stroke falls rapidly with time following a TIA, ${ }^{3-7}$ and that there are no published data on the long terms risks of stroke and other vascular evens in patients who survive event-free for a number of years, it is uncertain how aggressive the preventive treatment should be, and for how long it should be continued. Current clinical guidelines and recent reviews give no specific advice on this. ${ }^{9-13}$

We aimed to determine the 10 year risks of stroke, myocardial infarction, and vascular death in a cohort of patients with a past history of a non-recent TIA. These patients would generally be regarded as "low risk" because they were already beyond the early high risk period for recurrent stroke and had survived stroke-free for several months or years.

\section{METHODS}

We identified 290 eligible TIA patients at the end of planned follow up of the Oxfordshire community stroke project (OCSP), ${ }^{74}$ and a contemporaneous Oxford cohort study of hospital referred patients with TIA. ${ }^{15}$ Both cohorts had been assembled by the same original investigators, using the same diagnostic criteria. The OCSP was a population based study of the incidence of first ever TIA in a population of 105000 in Oxfordshire, UK, that ran from 1981 to 1986. The hospital referred cohort was recruited during the same period from patients referred from the remaining population of Oxfordshire that was not covered by the OCSP and included both first ever and recurrent TIA. Both studies involved a consecutive series of patients.

Patients were included in our study if they had presented with a TIA in either of the two original studies, and if they were alive and had not had a stroke at the end of 1988. Median time from most recent TIA to study entry was 3.8 years (interquartile range, 2.2 to 5.8 years). After ethical approval and permission from their general practitioner, patients were interviewed by a research nurse at home to ensure that they remained free of stroke, to record their current baseline clinical characteristics, and to take a blood sample for biochemical and haematological tests. 


\begin{tabular}{|c|c|c|}
\hline Characteristic & $N$ (median) & Per cent (range) \\
\hline Age (years) & (69) & (36 to 103 ) \\
\hline Male & 179 & 61.7 \\
\hline Diastolic blood pressure (mm Hg) & (82) & (50 to 117$)$ \\
\hline Systolic blood pressure $(\mathrm{mm} \mathrm{Hg})$ & (151) & (85 to 220 ) \\
\hline History of hypertension & 130 & 48.5 \\
\hline Antihypertensive treatment & 114 & 39.3 \\
\hline Previous myocardial infarct & 42 & 14.5 \\
\hline Treated diabetes & 15 & 5.2 \\
\hline Body mass index $\left(\mathrm{kg} / \mathrm{m}^{2}\right)$ & (24.9) & (15.6 to 45.9 ) \\
\hline \multicolumn{3}{|l|}{ Smoking } \\
\hline Never & 69 & 23.8 \\
\hline Ex-smoker & 153 & 52.8 \\
\hline Current & 68 & 23.4 \\
\hline Aspirin & 142 & 49.0 \\
\hline Total cholesterol (mmol/l) & (5.58) & (3.13 to 8.94$)$ \\
\hline $\mathrm{HDL}(\mathrm{mmol} / \mathrm{l})$ & $(1.17)$ & $(0.55$ to 3.02$)$ \\
\hline LDL (mmol/l) & (3.27) & (0.72 to 7.22$)$ \\
\hline
\end{tabular}

HDL, high density lipoprotein; LDL, low density lipoprotein.

Fatal events during follow up were identified through the Office for National Statistics National Health Service Central Register (ONS NHSCR). The ONS NHSCR coded the underlying cause of death according to ICD-9 and ICD-10. The main outcomes of interest were stroke (ICD-9: 430-438), myocardial infarction/coronary heart disease (ICD-9: 410414), and cardiovascular death (ICD-9: 390-459). Case notes of all subjects who died were also reviewed centrally. Non-fatal events were identified by direct contact with the family physician of all patients in 1994 and 1998. Non-fatal strokes were defined as events lasting more than 24 hours. Myocardial infarction was defined on the basis of changes in ECG and enzymes. The date of last mortality follow up was August 2000.

\section{Statistical analysis}

Kaplan-Meier survival analysis was used to calculate event rates. All analyses were limited to 10 years of follow up-that is, the date of last follow up for non-fatal events in 1998. For fatal events, the expected age and sex standardised death rates were estimated using mortality records for England and Wales. Differences between observed and expected death rates were expressed as standardised mortality ratios (SMR), with confidence intervals and probability $(p)$ values obtained from the Poisson distribution.

\section{RESULTS}

Just under half of the patients in the two original cohorts were eligible for inclusion in the current study: 89/184 (48\%) in the OCSP and 201/469 (43\%) in the hospital referred series. The main reasons for non-inclusion were that the patient had died before 1988, or that they had had a stroke.

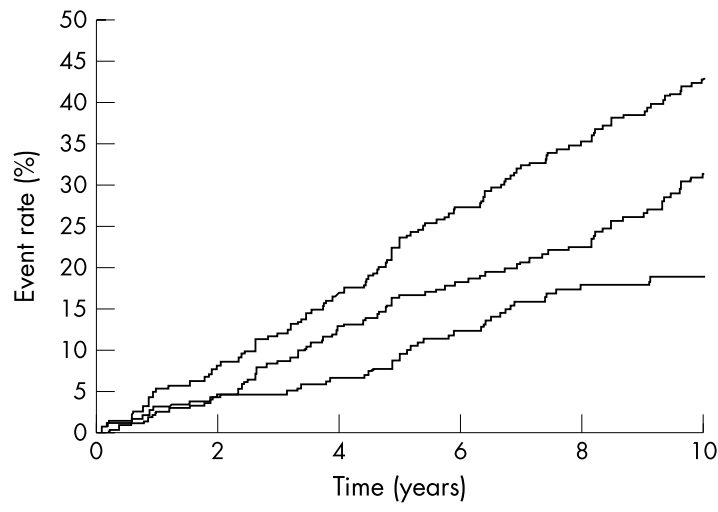

No of patients remaining

$\begin{array}{lllllll}\text { Stroke: } & 290 & 264 & 230 & 196 & 164 & 136\end{array}$

Stroke, Ml, $290 \quad 260 \quad 225 \quad 189 \quad 157 \quad 130$ or vasc death

Figure 1 Kaplan-Meier event rates for any stroke, any myocardial infarction, or death from coronary heart disease (MI/CHD), and any stroke, myocardial infarction, or vascular death.

The characteristics of the 290 study participants are described in table 1 . The cohort was elderly (median age 69 years), and predominantly male $(62 \%)$. The 89 patients derived from the OCSP were older than the 201 patients from the hospital referred cohort (mean age $71.9 v 68.2$ years, $p=0.005$ ), but there were no other significant differences in baseline characteristics. Ten year follow up was available in all patients.

\section{Fatal events}

During 10 years of follow up (2303 person-years) we observed 147 deaths, of which 20 were attributable to stroke (two with intracerebral haemorrhages) and 53 to myocardial infarction or coronary heart disease. In all, 87 deaths were caused by vascular disease. There were 60 non-vascular deaths. All cause mortality was not significantly greater than expected on the basis of age and sex specific national death rates (table 2 ). The number of stroke deaths was only slightly greater than expected $(\mathrm{SMR}=1.23 ; 95 \%$ confidence interval $(\mathrm{CI})$, 0.75 to $1.91 ; \mathrm{p}=0.4$ ), but the number of deaths from myocardial infarction or coronary heart disease was significantly higher than expected $(\mathrm{SMR}=1.47 ; 95 \% \mathrm{CI}, 1.10$ to $1.93 ; \mathrm{p}=0.009$ ).

\section{All vascular events}

Overall, 45 subjects had a non-fatal or fatal stroke and 67 had a non-fatal or fatal myocardial infarction or died from coronary heart disease. Kaplan-Meier curves for any stroke, any myocardial infarction or coronary heart disease, and any

Table 2 Comparison of observed death patterns and those expected using age/sex standardised rates

\begin{tabular}{lllll}
\hline \multirow{2}{*}{ Cause of death } & \multicolumn{2}{l}{ All patients $(\mathrm{N}=290)$} & \\
\cline { 2 - 3 } & Observed & Expected* & SMR (95\% Cl) & p Value \\
\hline All cause & 147 & 140.1 & $1.05(0.89$ to 1.23$)$ & 0.57 \\
Cancer & 22 & 32.7 & $0.67(0.42$ to 1.02$)$ & 0.06 \\
Respiratory & 21 & 23.2 & $0.91(0.56$ to 1.38$)$ & 0.75 \\
Stroke & 20 & 16.2 & $1.23(0.75$ to 1.91$)$ & 0.40 \\
MI/CHD & 53 & 36.0 & $1.47(1.10$ to 1.93$)$ & 0.009 \\
Other vascular & 14 & 12.2 & $1.15(0.63$ to 1.93$)$ & 0.68 \\
Other & 17 & 19.9 & $0.85(0.50$ to 1.37$)$ & 0.61 \\
\hline
\end{tabular}

*Based on age and sex standardisation using official England and Wales mortality data $\mathrm{CHD}$, coronary heart disease; $\mathrm{Cl}$, confidence interval; $\mathrm{Ml}$, myocardial infarction. 
stroke, myocardial infarction, or vascular death are shown in fig 1 . There were no significant differences in any of these risks between subjects derived from the OCSP and subjects derived from the concurrent hospital referred cohort. For each outcome, event rates were constant through time. The 10 year risk of any stroke was $18.8 \%$ (95\% CI, $13.6 \%$ to $23.7 \%$; 45 events) and the 10 year risk of myocardial infarction or death from coronary heart disease was $27.8 \%$ (95\% CI, $21.8 \%$ to $33.3 \%$; 67 events). In all, 114 patients had at least one major vascular event (stroke, myocardial infarction, or vascular death), giving a 10 year risk of any first stroke, myocardial infarction, or vascular death of $42.8 \%$ (95\% CI, $36.4 \%$ to $48.5 \%$ ).

\section{DISCUSSION}

There have been three previous studies of long term outcome after TIA, ${ }^{16-18}$ and one study in patients with minor stroke. ${ }^{19}$ However, all of these studies recruited patients within days or weeks of the presenting event and therefore include the early period of follow up during which the stroke risk is high. Two studies were retrospective. ${ }^{16}{ }^{17}$ One of these was confined to patients under 40 years of age ${ }^{17}$ and the other was done in the 1960s and early 1970s before routine use of antiplatelet agents and other preventive treatments. ${ }^{16}$ The one prospective study with long term follow up after TIA was based on only 18 patients. ${ }^{18}$

Thus our prospective study is the only large one of long term prognosis after a TIA in the modern era. It is also the first published study of the long term risks of stroke, myocardial infarction, and vascular death in patients who have survived free of stroke for several months or years after a TIA. It provides useful data on which clinical decisions about prevention of stroke and other vascular events can be based. We have shown that although the risk of stroke is relatively low in patients who survive the early high risk period, the risk of coronary vascular events remains high, and the overall risk of major vascular events is constant at about $4 \%$ per year.

The cost-effectiveness of vascular preventive treatmentssuch as new antiplatelet drugs, antihypertensive drugs, and statins-depends on the absolute risk of major vascular events. ${ }^{20}$ The $4 \%$ combined annual risk of stroke, myocardial infarction, or vascular death in our cohort did not diminish with time, at least out to 10 years of follow up. Thus even in patients who had remained free of major vascular events for over 10 years after a TIA, the overall vascular risk remains clinically significant. In the absence of evidence of reduced effectiveness of preventive treatments with increasing age or length of treatment, our data suggest that such treatments should be continued for at least 10 years after a TIA, and possibly for life.

Although we have shown that the long term vascular risk is high in patients with a non-recent TIA, these risks are probably underestimates for two reasons. First, our eligibility criteria meant that we had a relatively low risk cohort. We excluded any patient who had had a stroke during follow up in the original studies up to 1988. These patients would have had a higher risk of further strokes, and possibly of other vascular events, during further follow up than those who had remained stroke-free. Second, it is possible that ascertainment of non-fatal events may have been less effective than for fatal events because we relied to a significant extent on ascertainment of events from the medical records of the family physician. However, if our cohort was "low risk" and we did miss some non-fatal vascular events, then this would only further emphasise our overall conclusion that the long term vascular risk remains high in patients with a non-recent TIA.

Our study does, however, have various other shortcomings. First, in order to achieve a sufficient sample size we studied a cohort that was derived from two original studies, one community based and one hospital based. However, although this is not ideal, both cohorts had been assembled by the same investigators from the same geographical region using the same diagnostic criteria. Moreover, we applied the same eligibility criteria to both studies, and both cohorts were at the same time point in the evolution of their disease (mean of four years since the last TIA). Consequently, with the exception of a small difference in age, there were no significant differences between the cohorts in baseline clinical characteristics, and we found no differences in the risks of vascular events on subsequent follow up. Indeed, this lack of heterogeneity, despite the differences in origin of our patients, supports the generalisability of our findings. Second, we did not have sufficient numbers of strokes for a reliable determination of the risk of vascular events separately in the different pathological and aetiological subtypes of TIA, or of risk in relation to particular baseline clinical characteristics.

Limitations aside, this is the first long term follow up study of patients with TIA in the modern era when treatment of hypertension and hyperlipidaemia and the use of antiplatelet agents are likely to have improved prognosis. Despite this, we found that the combined risk of first stroke, myocardial infarction, or vascular death was approximately $4 \%$ a year and did not diminish with time. These results suggest that it is important to continue vascular preventive strategies in the long term in patients with a previous TIA, even if they have been free of any major vascular events for several years. In routine clinical practice it is likely that the risks will be higher than those reported here, and it is unlikely that they will be any lower in patients with a previous stroke rather than a TIA. The requirement of the National Service Framework for Older People that all general practices in the United Kingdom set up registers of patients with a previous TIA or stroke ${ }^{8}$ therefore appears to be reasonable, and should facilitate the identification of patients in whom there is scope for more active preventive treatment.

\section{ACKNOWLEDGEMENTS}

Barbara Morgan collected the baseline data. Sarah Matthews and Jean Harcourt collected data on non-fatal outcomes. We thank Charles Warlow for allowing us access his TIA cohorts after completion of his initial studies. This study was supported by the Wellcome Trust (project grant number 18365/1.5) and the NHS Executive South East (SPGS 753). TGC holds an NHS (UK) Research Training Fellowship (RTF 098). MFGM is funded by Cancer Research UK, and PMR is funded by the Medical Research Council.

\section{Authors' affiliations}

T G Clark, Centre for Statistics in Medicine, University of Oxford, Oxford M F G Murphy, Cancer Research UK General Practice Research Group, Institute of Health Sciences, Oxford

P M Rothwell, Stroke Prevention Research Unit, University of Oxford, Radcliffe Infirmary, Oxford

Competing interests: none declared

\section{REFERENCES}

1 Dippel D, Koudstaal PJ. We need stronger predictors of major vascular events in patients with a recent TIA or non-disabling stroke. Stroke 1997;28:774-6.

2 Rothwell PM, Warlow CP, on behalf of the European Carotid Surgery Trialists' Collaborative Group. Prediction of benefit from carotid endarterectomy in individual patients: a risk modeling study. Lancet 1999;353:2105-10.

3 Hankey GJ, Slattery JM, Warlow CP. Transient ischaemic attacks: which patients are at high (and low) risks of serious vascular events? J Neurol Neurosurg Psychiatry 1992;55:640-52

4 Howard G, Toole JF, Frye-Pierson J, et al. Factors influencing the survival of 451 transient ischaemic attack patients. Stroke 1987;18:552-7.

5 Dutch TIA Trial Study Group. Predictors of major vascular events in patients with a transient ischaemic attack or nondisabling stroke. Stroke 1993;24:527-31

6 Kernan WN, Viscoli CM, Brass LM, et al. The Stroke Prognosis Instrument II (SPI II): a clinical prediction instrument for patients with transient ischaemia and non-disabling ischaemic stroke. Stroke 2000;31:456-62. 
7 Dennis M, Bamford J, Sandercock $P$, et al. Prognosis of transient ischemic attacks in the Oxfordshire Community Stroke Project. Stroke 1990;21:848-53.

8 Department of Health. National service framework for older people. London: Department of Health, 2001. (www.doh.gov.uk/nsf)

9 Scottish Intercollegiate Guidelines Network. SIGN guidelines: management of patients with stroke. Edinburgh: SIGN, 1997 (www.sign.ac.uk)

10 Feinberg WM, Albers GW, Barnett HJM, et al. Guidelines for the management of transient ischaemic attacks. From the ad hoc committee on guidelines for the management of transient ischaemic attacks of the Stroke Council of the American Heart Association. Stroke 1994; 25: 1320-35

11 Wolf PA, Clagett GP, Easton JD, et al. Preventing ischemic stroke in patients with prior stroke and transient ischemic attack: a statement for healthcare professionals from the Stroke Council of the American Heart Association. Stroke 1999;30:1991-4.

12 Warlow CP, Dennis MS, van Gijn J, et al. Preventing recurrent stroke and other serious vascular events. In: Stroke: a practical guide to management. Oxford: Backwell Science, 2001:653-722.
13 Gubitz G Sandercock P. Prevention of ischaemic stroke. BM 2000;321:1455-9.

14 Dennis MS, Bamford JM, Sandercock PA, et al. Incidence of transient ischemic attacks in Oxfordshire, England. Stroke 1989;20:333-9.

15 Hankey GJ, Slattery JM, Warlow CP. The prognosis of hospital-referred transient ischaemic attacks. J Neurol Neurosurg Psychiatry $1991 \cdot 54: 793-802$

16 Muuronen A, Kaste M. Outcome of 314 patients with transient ischaemic attacks. Stroke 1982;13:24-31.

17 Larsen BH, Sorensen PS, Marquardsen J. Transient ischaemic attacks in young patients: a thromboembolic or migrainous manifestation. A 10 year follow-up study of 46 patients. J Neurol Neurosurg Psychiatry 1990:53:1029-33

18 Ueda K, Kiyohara Y, Hasuo Y, et al. Transient cerebral ischaemic attacks in a Japanese community, Hisayama, Japan. Stroke 1987; 18:844-8.

19 Prencipe $M$, Culasso F, Rasura $M$, et al. Long-term prognosis after a minor stroke. Stroke 1998:29:126-32.

20 Hankey GJ. Impact of treatment of people with transient ischaemic attacks on stroke incidence and public health. Cerebrovasc Dis 1996;6(suppl 1):26-33

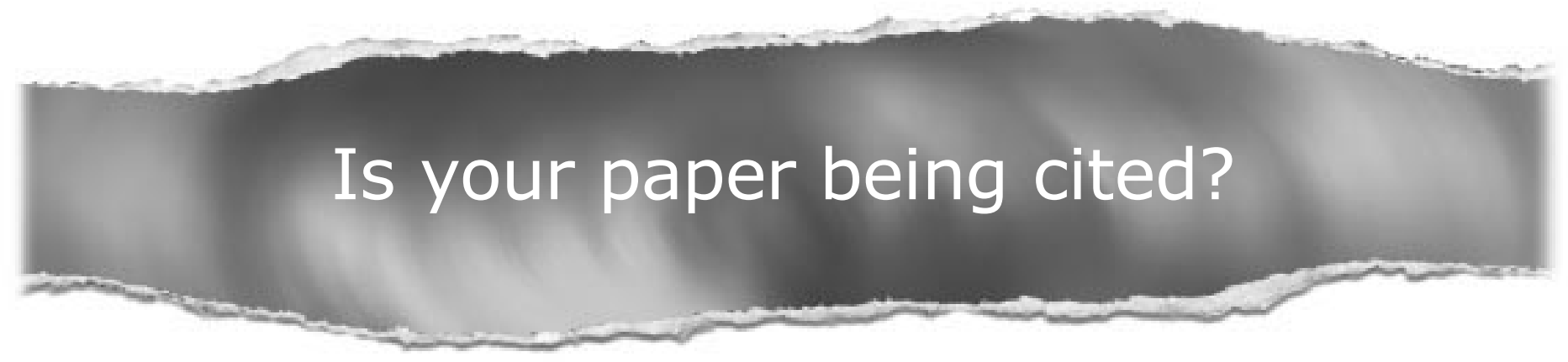

\section{CiteTrack service}

CiteTrack will alert you by email whenever new content in the Journal of Neurology, Neurosurgery, and Psychiatry or a participating journal is published that matches criteria you want to track Topics: Tell CiteTrack which words or subjects to watch for in new content Authors: Be alerted whenever key authors you are following publish a new paper Articles: Know whenever a paper of interest to you is referenced by another paper

\section{www.jnnp.com}

